\title{
Cloud Computing Software Testing Research Advancements
}

\author{
Nirmal Kumar Gupta \\ Jaypee University Anoopshahr \\ CSE Department \\ Anoopshahr
}

\author{
Mohammad Qasim Rafiq \\ Jaypee University Anoopshahr \\ CSE Department \\ Anoopshahr
}

\begin{abstract}
The emergence of cloud computing has brought tremendous impact on software organizations and software architecture design. The new environment requires software testing methods, technologies and tools to be changed. This research summarizes the contents of software testing in cloud computing environment. The definition, characteristics, types and scope of cloud computing software testing are discussed in presented research. Discussion of software testing services related research work is performed. Finally, it points out some issues that should be studied deeply in software testing in cloud computing environment.
\end{abstract}

\section{General Terms}

Software Testing, Cloud Computing

\section{Keywords}

Software Testing, Cloud Computing, Cloud Testing, TaaS, SaaS, PaaS

\section{INTRODUCTION}

In recent years, the emergence of cloud computing technology to the software development organizations and software architecture designers has brought great impact. Software as a Service (SaaS), Platform as a Service (PaaS) and Infrastructure as a Services (IaaS) have become the basic service models of cloud computing. The emergence of these service models have changed the development and utilization of software products, therefore it requires that the software testing methods, techniques and tools should also be changed.

The cloud-computing environment differs from the conventional development environment therefore the software testing process also has to be specific accordingly.

To address the software testing issues related to cloud computing environment many researchers are working in academia and industry, but this research is still in its infancy.

This paper aims to track and summarize the latest research and application of software testing in cloud computing environment. It is hoped that more scholars and technicians will understand and focus on cloud computing software development, in order to promote software testing in cloud computing more in-depth and for wide range of applications.

The main idea of Cloud Computing was first introduced very earlier during 1960's by John McCarthy when he pointed out that "computation may someday be organized as a public utility" in his book [30]. But the term 'Cloud Computing' was first used by companies Google and IBM during 2007 for the services which could be provided over web for accessing software, computing and files instead of local desktop machines [31][32]. In 2011 The National Institute of Standards and Technology (NIST) has proposed a basic concept of cloud computing that defines cloud computing as a model that allows the sharing of many computing resources as services to various clients [33]. Since 2010, various researchers started to pay attention to consider software testing as 'Testing as a Service' (TaaS) in cloud computing paradigm. Various research papers were published considering different aspects of this subject.

Incki et al. [29] provided an overview regarding main contributions, trends, gaps, opportunities, challenges and possible research directions for cloud computing software testing. They provided a review of software testing over the cloud literature and categorize the body of work in the field. Gao, Jerry, et al. [34] in their research provides a comprehensive review and tutorial on cloud-based TaaS for SaaS applications. Their work offers some essential concepts on TaaS definitions, scope, motivations and benefits, as well as classified testing services and test environments. RiunguKalliosaari, Leah, et al. [35] performed a qualitative study, which addresses the adoption, utilisation and effects of cloudbased testing in different organizational contexts. They used the results of their study to propose a strategy that could be used to assist practitioners in their decision-making processes towards adoption of cloud-based testing.

The rest of this paper is structured as follows. The next section presents the basic definitions and characteristics of cloud computing software testing. Section 3 discusses the research scope and classification of cloud computing software testing based on various criterion. Section 4 is focused on testing service research involving various quality models and other aspects. Finally, the conclusion remarks are given in Section 5 .

\section{DEFINITIONS AND CHARACTERISTICS}

\subsection{Definitions of cloud computing software testing}

The research in cloud computing software testing is still going on and still in infancy, the basic concepts of interpretations by the different scholars are not the same, and here the following representative definitions are described:

Definition 1: Cloud Testing is a form of software testing in which web applications that control Cloud Computing environments try to find to simulate real world user traffic as a mean of load testing and stress testing web sites [1].

Definition 2: Cloud-based software testing [2] refers to the use of cloud technology and solutions for testing and measurement activities in the cloud-computing environment.

Definition 3: There are two levels in which cloud computing can be defined [3]. The first level definition includes the 
services, which use the existing cloud-computing environment to provide the service resources, more realistic generation of simulated user load, fast and efficient load, and Stress testing; the second level of definition refers to the construction of cloud computing infrastructure services and the higher-level cloud computing applications testing.

In the definition of three different documents, Definition 1 emphasizes the use of cloud computing resources to simulate user traffic, and Definition 2 emphasizes the use of cloud computing environments and solutions for software testing activities. Definition 3 combines the previous two definitions to better reveal the characteristics and nature of cloud computing and software testing. The shortcomings of the above three definitions are not effectively identified in terms of test behavior and test outsourcing services in cloud computing software testing. Here in this paper we divide the concept of cloud test into two levels: specific and generalized, from the point of view of whether there is a third party test service. Now we can give the new definition as follows:

Definition 4: specific cloud computing software testing refers to use cloud-computing platform by the software developers as the on demand infrastructure to obtain the required test resources to complete a variety of test tasks of software testing activities. The generalized cloud computing software test refers to the delivery and use of the Testing as a Service (TaaS), which is used by the test service provider to provide the third party software testing services to the software testers, including the functional testing, test maintenance, test support and other test services.

\subsection{The Characteristics of Cloud Computing Software Testing}

Various new technologies and innovative service models have been proposed in the literature which use different testing techniques for cloud computing and that differentiates them from traditional software testing. Parveen et al. [4] discussed the advantages and different issues of performing unit testing, automated testing, performance testing and other testing activities in cloud computing environment based on their own practical experience. Ciortea et al [5] discussed the cloud computing software testing to reduce costs, reduce complexity, dynamic allocation of resources in three characteristics aspects through the Cloud9 platform application. Oriol et al. [6] summarized the advantages of cloud platform software testing in terms of performance and security through the deployment of the YETI project in cloud computing platforms, and suggested that virtual machine applications would help to reduce the security limitations of test code. Riungu et al. [7] used the rooting theory to describe the benefits of cloud computing to software testing from the perspective of cost, flexibility, market, through interviews with software testing service providers and test users. Their research involved test data security, resource availability, management and other aspects of cloud computing services. Candea et al. [8], Kalliosaari et al. [9], Yang et al. [10] also summarized the characteristics of cloud testing from the aspects of technology, price, quality of service and so on. P. Jamshidi et al. [28] tackled the problem of dynamic allocation of resources for cloud-based applications facing unpredictable workloads to decrease cost of ownership without violating SLAs. Based on the above literature work, we compare the advantages of cloud computing software test and traditional software test mode as following: a) cost savings. With the ever-changing needs of small and medium-sized businesses, testing requires the constant deployment of new devices and the use of cloud models, providing a test environment that can be deployed and configured in the cloud, dramatically reducing cost.

b) Real simulation. Cloud testing can more truly simulate the distributed virtual user environment, including geographical location, browser, operating system, network bandwidth and other features, to avoid the simulation of a single user form.

c) Available on request. Test process, do not understand the test requirements, resulting in a great waste of test resources, and cloud testing provides a test-on-demand approach; the user can be flexible and on-demand deployment of test resources, environment, when the test can be released after the release of related resources etc. can be performed.

d) Early testing. To improve efficiency load, stress and other tests can be carried out in the early stages of development as soon as possible, without the last unit, integration, system.

e) Accelerated testing. When the test case is very large or repeatedly run the test, then the workload will be very large. In such cases, the "cloud" can be used to run test cases concurrently to speed up the testing process.

f) Regulatory standards. Cloud testing provides a common solution for testing different application systems, with the ability to pre-define a variety of standards for testing the desired environment. In a sense, this will inevitably promote the standardization of different application systems, thus accelerating the development of cloud technology.

\section{RESEARCH SCOPE AND CLASSIFICATION OF CLOUD COMPUTING SOFTWARE TESTING \\ 3.1 Research Scope of Cloud Computing Software Testing}

Gao et al. [2] summarize the research scope of cloud computing software testing. The scope of cloud computing software testing is shown in Fig 1. The scope of their study can be divided into three levels:

(1) The major study in current literature at first level of the cloud-computing platform is focused on the cloud platform IaaS layer of test research which includes the ability to build cloud computing infrastructure services testing platform itself. Tan et al. [11] detected the problem of failure of the Hadoop node system log, and detected the execution of Hadoop more completely from the black box and the white box approaches [12]. Pan et al. [13] proposed the Ganesha method to determine the accuracy of the method by simulating common errors in Hadoop. Dean J. et al. [14] proposed MapReduce performance test method based on Phoenix - based Linux without recompiling application logic. These test methods provide a good reference to effectively improve the reliability of the cloud platform for other cloud test software.

(2) The study at second level of the cloud-computing platform is to use the cloud-computing platform to provide the resources of the various testing activities. Ganon et al [15] discussed the use of Amazon cloud platform for VoIP network management system test examples; effectively solve the problem of large-scale test environment. It is difficult to accurately test the performance of large-scale network management system. Baride et al. [16] discussed the application of cloud computing test platform in mobile 
development, and given the system structure of mobile test cloud platform. Qian L. et al. [17] proposed a new generation of telecom services based on cloud computing support system evaluation platform, and focused on the key functions and their implementation. This level of testing focuses on issues such as cloud testing technology, cloud platform, automated testing tools and cloud test project management technology etc.

(3) The third level mainly studies the related problems in TaaS. TaaS is a new service model for the software testing industry that supports the demand-oriented software testing services market where software organizations can access software testing services on demand. Candea et al. [8] limited TaaS to the cloud-based automated software testing service model, emphasizing the difference from traditional manual participation in service models. Sogeti believes that TaaS is an open, flexible and low-cost software testing solution based on a broad "service" that divides it into Level 1 and Level 2 [18]. King et al. [19] referred to "Autonomic Self-Testing" (AST) and "Test Support as a Service" (TSaaS), which have ability to strengthen the self-test process and designed to be processed through the cloud computing platform. Gaisbauer et al. [20] proposed the Virtualized-aware Automated Test Service (VATS) test model, which provides test services using virtual devices on the cloud-computing platform. TaaS is considered a key but difficult problem in the research of cloud computing software. It is basically involved in cloud computing, software testing and service outsourcing. It is regarded as a combination of information science, computer science and service science.

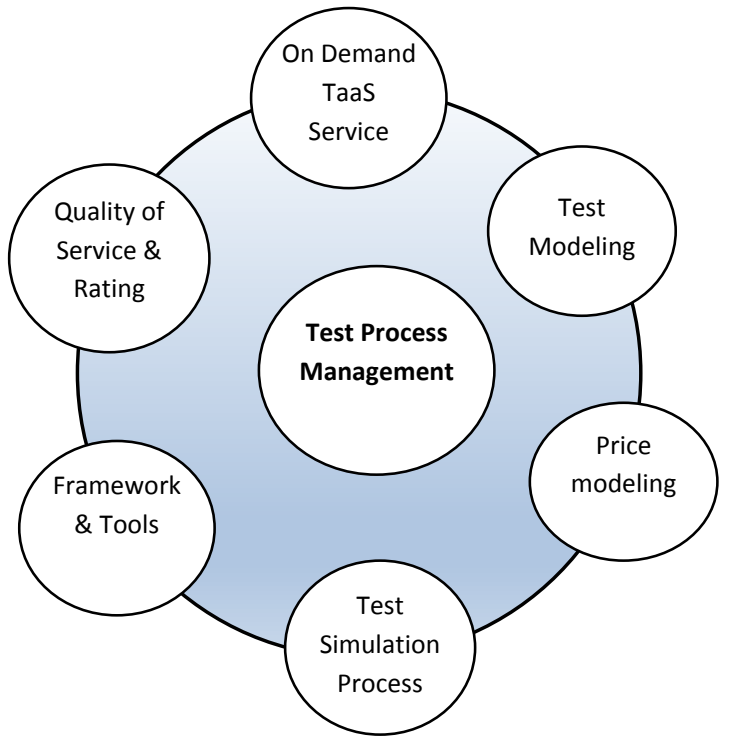

Fig 1: Cloud computing software test coverage map

\subsection{Classification of Cloud Computing Software Testing}

\section{(1) Classification based on test tasks}

It is the most intuitive classification method, literature [1], [3] and [21] are using this method to classify cloud tests. In this paper, we divide the cloud test into eight types: stress test, load test, performance test, function test, compatibility test, browser performance test, delay test and safety test, as shown in Fig 2.

The stress test in the cloud-computing environment refers to the large-scale computing power through the cloud platform to determine the performance bottleneck of a software system and obtain the maximum service level that the system can provide. Load testing is a type of performance testing used to examine the system behavior during normal and high loads and determines if the software system in the cloud-computing platform can handle high loads given a high demand of end users. Performance testing refers to use of cloud platform determine how a system performs in terms of responsiveness and stability under a particular workload. It can also serve to investigate, measure, validate or verify other quality attributes of the system, such as scalability, reliability and resource usage. Functional testing refers to testing the system functions of $\mathrm{SaaS}$ and non-SaaS software modules. Compatibility testing is the use of cloud computing platform for a variety of operating systems, a variety of databases and a variety of browser environment test software compatibility. Browser performance testing is to test the performance of SaaS and non-SaaS software in different browsers. A latency test is a test of delay response when the application is deployed on a cloud platform. Security testing is the test of SaaS and nonSaaS software system security.

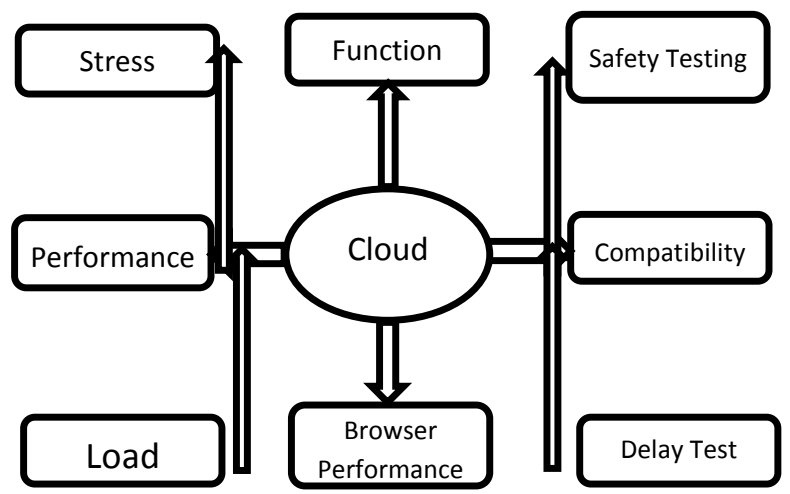

Fig 2: Testing and Classification of Cloud Computing Software Based on Test Task

\section{(2) Classification based on the test user} role

In the literature [3] the author proposed another classification method, from the perspective of cloud computing providers, users and system developers. According to the different cloud-computing environment, cloud computing software testing is divided into four types, such as Fig 3 shows.

i. Testing a SaaS in a Cloud: This type is a quality assurance for SaaS functionality and nonfunctional service requirements in the cloud.

ii. Testing of a Cloud: This type of testing is performed from an external perspective for quality validation of the functions and service features specified by the cloud, cloud providers, SaaS providers, and end users who are interested in this type of test.

iii. Testing within the cloud: This type of testing is performed from the internal perspective of the cloud infrastructure and specific cloud capabilities for quality checks. Only cloud providers can perform this type of test, because they have access including internal SaaS, automatic function, Security, management and monitoring of the internal infrastructure and connectivity functions.

iv. Testing over Clouds: This type of testing includes deployment of cloud-based service applications in the cloud, including private cloud, public cloud, mixed cloud system-level application services and is typically tested by cloud-based application's System vendor. 


\section{RESEARCH ON CLOUD COMPUTING SOFTWARE TESTING SERVICE}

Cloud computing software testing service research mainly involves service model, price model and service quality evaluation model and other aspects, the main research results are as follows:

\section{(1) Service model}

Some scholars have studied the theoretical problems of cloud computing software testing services, and constructed relevant theoretical models through graphs and other tools [22]. Chan et al. [23] constructed the cloud-computing model by graph theory, using the service resource as a node in the graph, using the use of the resource as the edge of the graph. Based on the cloud-computing model, the application test standards and algorithms have been put forward. Vengattaraman et al. [24] described the relationship between different cloud computing applications and external consumer services by studying the self-built software in the cloud-computing environment and constructed the initial model. Priaadarsini K. et al. [25] constructed the TaaS architecture model and service composition model.

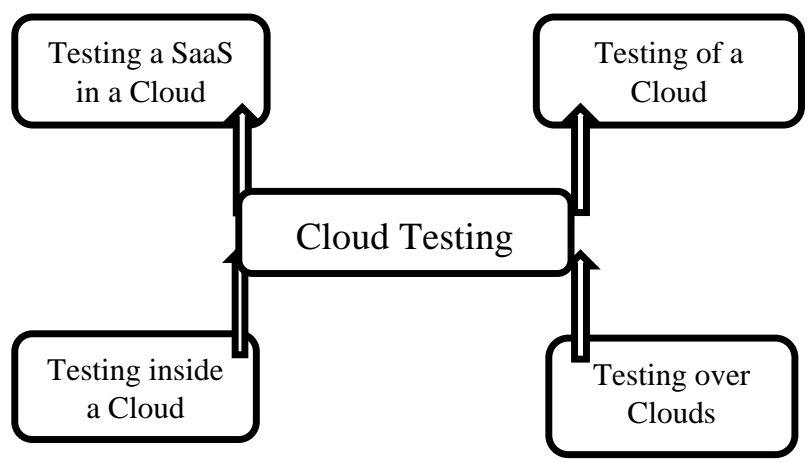

Fig 3: Software test classification based on the user role of cloud computing [2]

\section{(2) The price model}

At present, the general cloud computing platform, such as Amazon's EC2 [26] are based on the rental time of resources to charge, and cloud testing services billing method is not necessarily suitable for this approach. Candea et al. [8] suggests that cloud computing software testing should be more concerned about the long term users, from the economies of scale gains. Ciortea et al [5] from the Cloud9 project reality, that the cloud computing software testing has a certain degree of particularity, the charging method should be decided based on the user's test objectives. For example, if the test target can be prorated into a coverage level, the user can pay based on the percentage of the coverage level; if the goal is to find the cause of the crash, the user can pay for the number of reasons found. It can be believed that the charging model than just in accordance with the use of resources to pay is more reasonable, to encourage users to use more testing services, thereby improving the reliability of software quality.

\section{(3) Service quality evaluation model}

Software testing activities can be viewed as a service provided by the testers to software developers and end users regarding error minimization, software verification and software validation [27]. Riungu et al. [7] mentioned the importance of good quality of service for improving cloud computing software testing. Yang et al. [10] considered that "software testing as a service" was a collection of two services provided by software testers to developers and end users, defined as Service I and Service II, using the SERVQUAL model to construct a cloud computing platform Evaluation Model of Software Testing Service Quality. The model has some innovation, but the model does not construct a quality evaluation scale.

\section{CONCLUSION}

In general, cloud-computing software testing related research is still in its infancy, has not yet formed a sound theoretical system. It is necessary to study the construction of cloud computing software testing tools and platforms, test process management, multi-user collaborative test integration method, test data security management technology, price model and more on the basis of existing research. Other studies to perform include service description, the impact of software quality and software management to bring the changes and other issues, and the development of relevant technical standards, service standards and safety management standards.

\section{REFERENCES}

[1] Prakash V. and Gopalakrishanan S. 2012. "Cloud Computing Solution-Benefits and Testing Challenges", Journal of Theoretical and Applied Information Technology, Volume 39, Number 2, 2012.

[2] Gao J., Bai X. and Tsai W. 2011. "Cloud Testing- Issues, Challenges, Needs and Practice", Software engineering: an international Journal (SeiJ), Volume 1, Number 1, September 2011.

[3] Mladen A Vouk. 2008. "Cloud computing: Issues, research and implementations". ITI 2008 30th International Conference on Information Technology Interfaces, Volume 16, Number 4, pp. 31-40, 2008.

[4] Parveen T. and Tilley S. 2010. "When to Migrate Software Testing to the Cloud?". Third International Conference on Software Testing, Verification, and Validation Workshops, Paris, pp. 424-427.

[5] Ciortea L., Zamfir C. and Bucur S. 2010. Cloud9: a software testing service. ACM SIGOPS Operating Systems Review, Volume 43, Number 4, pp. 5-10.

[6] Oriol M. and Ullah F. 2010. YETI on the Cloud. In Proceedings of the 2010 Third International Conference on Software Testing, Verification, and Validation Workshops (ICSTW '10). IEEE Computer Society, Washington, DC, USA, pp. 434-437.

[7] Riungu L.M., Taipale O. and Smolander K. 2010. Software Testing as an Online Service: Observations from Practice. In Third International Conference on Software Testing, Verification, and Validation Workshops (ICSTW), pp. 418-423.

[8] Candea G., Bucur S. and Zamfir C. 2010. Automated software testing as a service. In ACM SOCC.

[9] Kalliosaari L.R., Taipale O., Smolander K. and Richardson I. 2016. Adoption and use of cloud-based testing in practice. Software Quality Journal, Volume 24, Number 2, pp. 337-364.

[10] Yang Y., Onita C., Dhaliwal J and Zhang X., 2009. "TESTQUAL: conceptualizing software testing as a 
service," Proc. 15th Americas conf. on information systems, San Francisco, California, USA, paper 608.

[11] Tan J., Pan X., Kavulya S., Gandhi R. and Narasimhan P. 2008. SALSA: analyzing $\operatorname{logs}$ as state machines. In

[12] Tan J., Pan X., Kavulya S., Gandhi R. and Narasimhan P. 2009. Mochi: visual log-analysis based tools for debugging hadoop. In Proceedings of the 2009 conference on Hot topics in cloud computing (HotCloud'09). USENIX Association, Berkeley, CA, USA.

[13] Pan X., Tan J., Kavulya S., Gandhi R. and Narasimhan P. 2010. Ganesha: blackBox diagnosis of MapReduce systems. SIGMETRICS Perform. Eval. Rev. 37, 3 (January 2010), pp. 8-13.

[14] Dean J. and Ghemawat S. 2008. MapReduce: simplified data processing on large clusters. Commun. ACM 51, 1 (January 2008), pp. 107-113.

[15] Ganon Z. and Zilbershtein I.E. 2009. Cloud-based Performance Testing of Network Management Systems. 2009 IEEE 14th International Workshop on Computer Aided Modeling and Design of Communication Links and Networks, Pisa, pp. 1-6.

[16] Baride S. and Dutta K. 2011. A cloud based software testing paradigm for mobile applications. SIGSOFT Softw. Eng. Notes 36, 3 (May 2011), pp. 1-4.

[17] Qian L., Luo Z., Du Y. and Guo L. 2009. Cloud computing: an overview. In: CloudCom'09: proceedings of the 1st international conference on cloud computing. Springer, Berlin, pp. 626-631.

[18] Candea G., Bucur S. and Zamfir C. 2010. Automated software testing as a service. In Proceedings of the 1 st ACM symposium on Cloud computing (SoCC '10). ACM, New York, NY, USA, pp. 155-160.

[19] King T.M. and Ganti A.S. 2010. Migrating Autonomic Self-Testing to the Cloud. In Proceedings of the 2010 Third International Conference on Software Testing, Verification, and Validation Workshops (ICSTW '10). IEEE Computer Society, Washington, DC, USA, pp. 438-443.

[20] Gaisbauer S., Kirschnick J., Edwards N. and Rolia J. 2008. VATS: Virtualized-Aware Automated Test Service. In Proceedings of the 2008 Fifth International Conference on Quantitative Evaluation of Systems (QEST '08). IEEE Computer Society, Washington, DC, USA, pp. 93-102.

[21] Tilley S., and Parveen T. 2012. Software Testing in the Cloud: Perspectives on an Emerging Discipline (1st ed.). IGI Global, Hershey, PA, USA.

[22] Lu H., Chan W.K. and Tse T.H. 2008. Testing pervasive software in the presence of context inconsistency resolution services. In Proceedings of the 30th international conference on Software engineering (ICSE '08). ACM, New York, NY, USA, pp. 61-70.

[23] Chan W.K., Mei L. and Zhang Z. 2009. Modeling and testing of cloud applications. IEEE Asia-Pacific Services
Proceedings of the First USENIX conference on Analysis of system logs (WASL'08). USENIX Association, Berkeley, CA, USA, pp. 6-6.

Computing Conference (APSCC), Singapore, pp. 111118.

[24] Vengattaraman T., Dhavachelvan P. and Baskaran R. 2010 Model of Cloud Based Application Environment for Software Testing. (IJCSIS) International Journal of Computer Science and Information Security, Volume 7, Number 3.

[25] Priyadarsini K, Balasubramanian V, Karthik S. 2011. Cloud Testing as a Service. Intematicnal Journal of Advanced Engineering and Technologies (IJAEST), Volume 6, Number 2, pp. 173 - 177.

[26] Armbrust M, Fox A, Griffith R, Joseph A, Katz R, Konwinski A, Lee G, Patterson D, Rabkin A, Stoica I, Zaharia M. 2010. A view of cloud computing. Communications of the ACM, Volume 53, Number 4, pp. 50-58.

[27] Bentley J. E, Bank W. 2005. NCC Software Testing Fundamentals - Concepts, Roles, and Terminology, In: Proceedings of SAS Conference, pp. 1 - 12.

[28] Jamshidi P., Ahmad A. and Pahl C. 2014. Autonomic resource provisioning for cloud-based software. In Proceedings of the 9th International Symposium on Software Engineering for Adaptive and Self-Managing Systems, Hyderabad, India, ACM, pp. 95-104.

[29] Incki, K., Ari, I., \& Sözer, H. 2012. A survey of software testing in the cloud. In IEEE Sixth International.Conference on Software Security and Reliability Companion (SERE-C), 2012 pp. 18-23.

[30] Parkhil F. Douglas, 1966. "The challenge of the Computer Utility.".

[31] Lohr S. 2007. "Google and IBM Join in "Cloud Computing'Research" New York Times 8.

[32] Vouk A, M. 2008."Cloud computing-issues, research and implementations", CIT. Journal of Computing and Information Technology, Volume 16, Issue 4, pp. 235246.

[33] Mell P. and Grance T., 2011. "The NIST definition of cloud computing [Recommendations of the National Institute of Standards and Technology-Special Publication 800-145]" NIST Special Publication, Volume 145 , pp. 7.

[34] Gao J., Bai X., Tsai W.T., and Uehara T. 2013.“Testing as a service (TaaS) on clouds", In Service Oriented System Engineering (SOSE), 2013 IEEE 7th International Symposium on (pp. 212-223). IEEE.

[35] Riungu-Kalliosaari L., Taipale O., Smolander K., and Richardson I., 2016. "Adoption and use of cloud-based testing in practice." Software Quality Journal, Volume 24, Issue 2, pp. 337-364. 